\title{
Anesthetic Management of a Patient with Hermansky-Pudlak Syndrome
}

\author{
Kelly Zach, Lopa Misra \\ Mayo Clinic Hospital, Phoenix, AZ, USA \\ Email: zach.kelly@mayo.edu
}

Received 28 January 2015; accepted 22 February 2015; published 26 February 2015

Copyright (C) 2015 by authors and Scientific Research Publishing Inc.

This work is licensed under the Creative Commons Attribution International License (CC BY). http://creativecommons.org/licenses/by/4.0/

c) (i) Open Access

\begin{abstract}
Hermansky-Pudlak Syndrome (HPS) is a rare genetic condition characterized by platelet function abnormalities and oculocutaneous albinism. Other systemic manifestations of the disease include pulmonary fibrosis, granulomatous colitis, impaired renal function, and cardiomyopathy. Due to the systemic extent of the disease, HPS has multiple anesthetic concerns and requires a detailed preoperative evaluation and close perioperative monitoring.
\end{abstract}

\section{Keywords}

\section{Hermanksy-Pudlak, Anesthesia}

\section{Introduction}

Hermansky-Pudlak Syndrome (HPS) is a rare genetic syndrome characterized by abnormal platelet aggregation and oculocutaneous albinism. Additional systemic complications of the disease include pulmonary fibrosis, granulomatous colitis, and impaired renal function. It is an autosomal recessive condition that is most commonly seen in northwest Puerto Rico where it has an incidence of 1 in 1800. The disorder often manifests in childhood with easy bruisability, epistaxis, or prolonged bleeding after surgery. The bleeding diathesis results from low levels of platelet granules required for appropriate platelet aggregation. Patients often have normal platelet levels but a prolonged bleeding time [1]. Given the tendency for excessive bleeding, particular considerations are required for HPS patients undergoing any surgical procedure. In addition, the systemic complications of the disease can present challenges for the anesthesiologist. With no cure, each organ system should be evaluated and optimized prior to surgery. Patients may require platelet transfusions. On the other hand, some patients are given prophylactic desmopressin preoperatively. All patients should be interrogated for the presence of respiratory involvement. A later and often fatal complication of the disease is progressive pulmonary fibrosis. Of the eight genotypic subtypes (HPS-1 to HPS-8), the first subtype (HPS-1) is the most common and also has the greatest 
propensity for development of pulmonary fibrosis [1]. As such, these patients are at risk for postoperative respiratory complications. Due to the systemic extent of the disease, HPS is an anesthetic concern requiring a precise preoperative examination and close perioperative monitoring. This case report describes a routine surgical procedure in a patient with HPS. We also offer a perioperative plan for patients with this condition, highlighting the management for the multiple anesthetic implications.

\section{Case Description and Review}

A 53-year-old female with a history of an overactive bladder presented for botox injection of the bladder. She underwent evaluation in the preoperative clinic revealing a medical history significant for Hermansky-Pudlak syndrome with an unknown subtype. Given the potential for systemic involvement of the disease, the patient's history was reviewed in detail to identify any anesthetic concerns. At the time of the procedure, the extent of the disease was limited to her eyes. She was legally blind with associated strabismus and horizontal nystagmus. She denied any bleeding tendencies including menometorrhagia, gingival bleeding, or epistaxis. She had undergone two previous urologic procedures before which she was prepped with the recommended dose of desmopressin (DDAVP). With these surgeries, she did not have any bleeding complications and did not require transfusions. On review of systems, the patient denied significant respiratory symptoms. Six months prior, she underwent pulmonary function tests revealing no obvious abnormalities with normal lung volumes, FEV1, FVC, and FEV1/FVC ratio. Additionally, multiple colonoscopies were clinically benign. Her laboratory data revealed normal blood counts and renal function. Following this evaluation, the patient was deemed appropriate for her urologic procedure.

Prior to her surgery, the patient was given a $0.3 \mathrm{mcg} / \mathrm{kcg}$ dose of desmopressin as recommended by hematology. General anesthesia was induced with propofol and fentanyl, and a laryngeal mask airway (LMA) was atraumatically inserted. Anesthesia was maintained with sevoflurane. The patient was placed in the dorsal lithotomy position and a cystoscope was introduced. The bladder was thoroughly evaluated without any remarkable findings. Subsequently 20 milliliters of 100 units of type A bladder botox was injected into the posterior bladder wall in a grid pattern. Following the injection, the bladder was inspected revealing no signs of active gross bleeding. The patient tolerated the procedure well, the LMA was removed, and she was recovered in the post-anesthesia care unit. Total blood loss for the procedure was less than 5 milliliters. She experienced no respiratory or bleeding complications and was discharged home with scheduled urology follow up.

\section{Discussion}

Despite the routine nature of this surgical procedure, this case of a patient with a rare genetic condition presented multiple anesthetic considerations. As mentioned, a hallmark of HPS is the platelet storage pool deficiency. Specifically, electron microscopy reveals low levels of dense platelet granules that are necessary for proper platelet aggregation [2]. Often, platelet levels are normal yet bleeding times can be prolonged. Patients presenting for surgery should undergo consultation with hematology due to the high risk for bleeding complications [3]. Platelet transfusions should be used to treat the bleeding diathesis associated with surgical procedures. Additionally, DDAVP has been shown to improve bleeding time in patients with Platelet function disorders [4]. It works by inducing an increase in plasma levels of von Willebrand factor (VWF) and coagulation factor VIII (FVIII) [5]. There remains limited data for the routine use of DDAVP for HPS patients undergoing surgery. In terms of bleeding time, patients with HPS have shown a variable response to DDAVP [6] [7]. Similarly, the prophylactic administration of DDAVP to a patient prior to subsequent obstetric deliveries resulted in inconsistent effectiveness [8]. Thus, review of the literature suggests that while DDAVP can be considered for prevention of perioperative bleeding, a consistent clinical response to its administration should not be implied. For patients with a poor response to DDAVP, recombinant factor VIIa (rFVIIa) can be considered. Case reports have demonstrated success in both the prevention and termination of bleeding complications in patients with HPS who receive rFVIIa [9] [10].

A later and often fatal complication of HPS is the development of rapid progressive pulmonary fibrosis, often resulting in premature death. The pathophysiologic mechanism is poorly understood, but is thought to result from deposition of ceroid as a consequence of lysosomal impairment. These ceroid deposits are believed to trigger an inflammatory response ultimately leading to fibrinogenesis and pulmonary fibrosis [11]-[13]. To date, drug therapy has proven largely unsuccessful. Pirfenidone, a novel medication with anti-inflammatory and anti- 
fibrotic properties has demonstrated slower progression of HPS in a select population [14]. In general, lung transplantation remains the only treatment option for patients with pulmonary fibrosis. There has only been one reported case of a successful lung transplant in a patient with HPS [15]. Due to the concern for respiratory decline in these patients, a thorough preoperative evaluation is essential. Functional status and exercise tolerance should be assessed. It is recommended that annual pulmonary function tests be performed and reviewed prior to surgery [3]. A restrictive lung pattern identifies the beginning of fibrotic development [12]. A decrease in forced vital capacity can identify worsening of the disease [11]. The risk for perioperative respiratory complications is an important consideration. Therefore, identification of declining respiratory status and optimization of function is especially critical in patients with HPS.

Additional complications of the disease should be evaluated. Renal impairment has been reported [13]. Furthermore, granulomatous colitis can be severe and refractory to corticosteroids and immunomodulators [16]. Cardiomyopathy has also been described in a small number of patients with HPS [17]. Although rare, these systemic manifestations can be life-threatening and are essential anesthetic considerations prior to any surgical procedure.

\section{Conclusion}

This case outlines the perioperative care of a patient with a rare genetic condition, Hermansky-Pudlak Syndrome (HPS). HPS is a systemic disease that results in platelet dysfunction and oculocutaneous albinism. Late manifestations include pulmonary fibrosis, renal insufficiency, and colitis. The widespread nature of the disease can produce unpredictable complications, particularly in patients undergoing surgical procedures. Therefore, potential anesthetic implications must be evaluated. Due to abnormal platelet aggregation, patients can have bleeding tendencies. Prophylactic desmopressin or platelet transfusions may be warranted. In addition, pulmonary fibrosis can lead to perioperative pulmonary complications. Particular attention should be paid to a patient's functional status. Pulmonary function tests can further evaluate a patient's respiratory reserve. Basic laboratory data can identify renal insufficiency. Overall, a detailed history and physical is important in these patients. A thorough preoperative evaluation allows for safe perioperative care in patients with this uncommon disease.

\section{References}

[1] Hurford, M.T. and Sebastiano, C. (2008) Hermansky-Pudlak Syndrome: Report of a Case and Review of the Literature. International Journal of Clinical and Experimental Pathology, 1, 550-554.

[2] Hazelwood, S., Shotelersuk, V., Wildenberg, S.C., Chen, D., Iwata, F., Kaiser-Kupfer, M.I., White, J.G., King, R.A. and Gahl, W.A. (1997) Evidence for Locus Heterogeneity in Puerto Ricans with Hermansky-Pudlaksyndrome. American Journal of Human Genetics, 61, 1088-1094. http://dx.doi.org/10.1086/301611

[3] Seward Jr., S.L. and Gahl, W.A. (2013) Hermansky-Pudlak Syndrome: Health Care throughout Life. Pediatrics, 132, 153160. http://dx.doi.org/10.1542/peds.2012-4003

[4] Coppola, A. and Di Minno, G. (2008) Desmopressin in Inherited Disorders of Platelet Function. Haemophilia, 14, 3139. http://dx.doi.org/10.1111/j.1365-2516.2007.01607.x

[5] Kaufmann, J.E. and Vischer, U.M. (2003) Cellular Mechanisms of the Hemostatic Effects of Desmopressin (DDAVP). Journal of Thrombosis and Haemostasis, 1, 682-689. http://dx.doi.org/10.1046/j.1538-7836.2003.00190.x

[6] Van Dorp, D.B., Wijermans, P.W., Meire, F. and Vrensen, G. (1990) The Hermansky-Pudlak Syndrome. Variable Reaction to 1-Desa-Mino-8D-Arginine Vasopressin for Correction of the Bleeding Time. Ophthalmic Paediatrics and Genetics, 11, 237-244. http://dx.doi.org/10.3109/13816819009020985

[7] Cordova, A., Barrios, N.J., Oritz, I., et al. (2005) Poor Response to Desmopressin Acetate (DDAVP) in Children with Hermansky-Pudlak Syndrome. Pediatric Blood \& Cancer, 44, 51-54. http://dx.doi.org/10.1002/pbc.20210

[8] Zatik, J. (2002) Variable Response of Hermansky-Pudlak Syndrome to Prophylactic Administration of 1-Desamino 8DArginine in Subsequent Pregnancies. European Journal of Obstetrics \& Gynecology and Reproductive Biology, 104, 165-166. http://dx.doi.org/10.1016/S0301-2115(02)00063-5

[9] Lohse, J., Gehrisch, S., Tauer, J.T. and Knöfler, R. (2011) Therapy Refractory Menorrhagia as First Manifestation of Hermansky-Pudlak Syndrome. Hamostaseologie, 31, S61-S63.

[10] Del Pozo Pozo, A.I., Jimenez-Yuste, V., Villar, A., Villar, A., Quintana, M. and Hernandez-Navarro, F. (2002) Successful Thyroidectomy in a Patient with Hermansky-Pudlak Syndrome Treated with Recombinant Activated Factor VII and Platelet Concentrates. Blood Coagulation \& Fibrinolysis, 13, 551-553. http://dx.doi.org/10.1097/00001721-200209000-00010 
[11] Pierson, D.M., Ionescu, D., Qing, G., Yonan, A.M., Parkinson, K., Colby, T.C. and Leslie, K. (2006) Pulmonary Fibrosis in Hermansky-Pudlak Syndrome. Respiration, 73, 382-395. http://dx.doi.org/10.1159/000091609

[12] Carter, B. (2012) Hermansky-Pudlak Syndrome Complicated by Pulmonary Fibrosis. Baylor University Medical Center Proceedings, 25, 76-77.

[13] Gahl, W.A., Brantly, M., Kaiser-Kupfer, M.I., Iwata, F., Hazelwood, S., Shotelersuk, V., Duffy, L.F., Kuehl, E.M., Troendle, J. and Bernardini, I. (1998) Genetic Defects and Clinical Characteristics of Patients with a Form of Oculocutaneous Albinism (Hermansky-Pudlak Syndrome). The New England Journal of Medicine, 338, 1258-1264. http://dx.doi.org/10.1056/NEJM199804303381803

[14] Gahl, W.A., Brantly, M., Troendle, J., Avila, N.A., Padua, A., Montalvo, C., Cardona, H., Calis, K.A. and Gochuico, B. (2002) Effect of Pirfenidone on the Pulmonary Fibrosis of Hermansky-Pudlak Syndrome. Molecular Genetics and Metabolism, 76, 234-242. http://dx.doi.org/10.1016/S1096-7192(02)00044-6

[15] Lederer, D.J. (2005) Successful Bilateral Lung Transplantation for Pulmonary Fibrosis Associated with the HermanskyPudlak Syndrome. The Journal of Heart and Lung Transplantation, 24, 1697-1699. http://dx.doi.org/10.1016/j.healun.2004.11.015

[16] Kouklakis, G., Efremidou, E.I., Papageorgiou, M.S., et al. (2007) Complicated Crohn’s-Like Colitis, Associated with Hermansky-Pudlak Syndrome, Treated with Infliximab: A Case Report and Brief Review of the Literature. Journal of Medical Case Reports, 1, 176. http://dx.doi.org/10.1186/1752-1947-1-176

[17] Huizing, M., Anikster, Y. and Gahl, W.A. (2000) Hermansky-Pudlak Syndrome and Related Disorders of Organelle Formation. Traffic, 1, 823-835. http://dx.doi.org/10.1034/j.1600-0854.2000.011103.x 
Scientific Research Publishing (SCIRP) is one of the largest Open Access journal publishers. It is currently publishing more than 200 open access, online, peer-reviewed journals covering a wide range of academic disciplines. SCIRP serves the worldwide academic communities and contributes to the progress and application of science with its publication.

Other selected journals from SCIRP are listed as below. Submit your manuscript to us via either submit@scirp.org or Online Submission Portal.
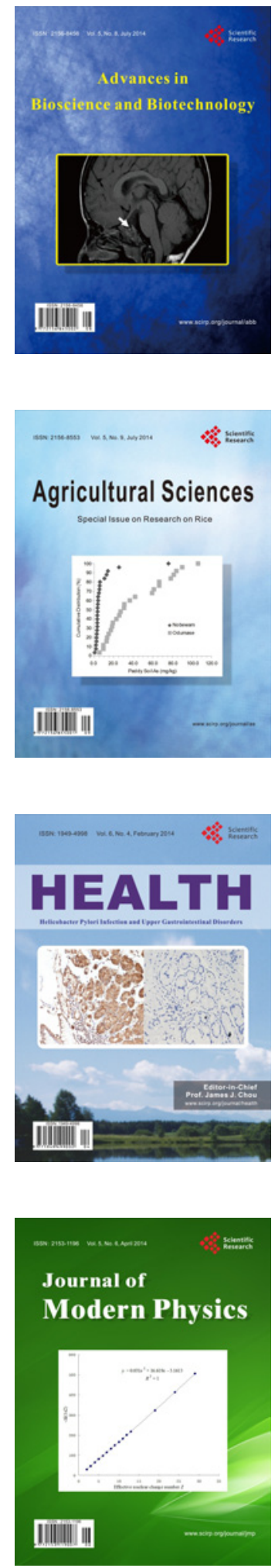
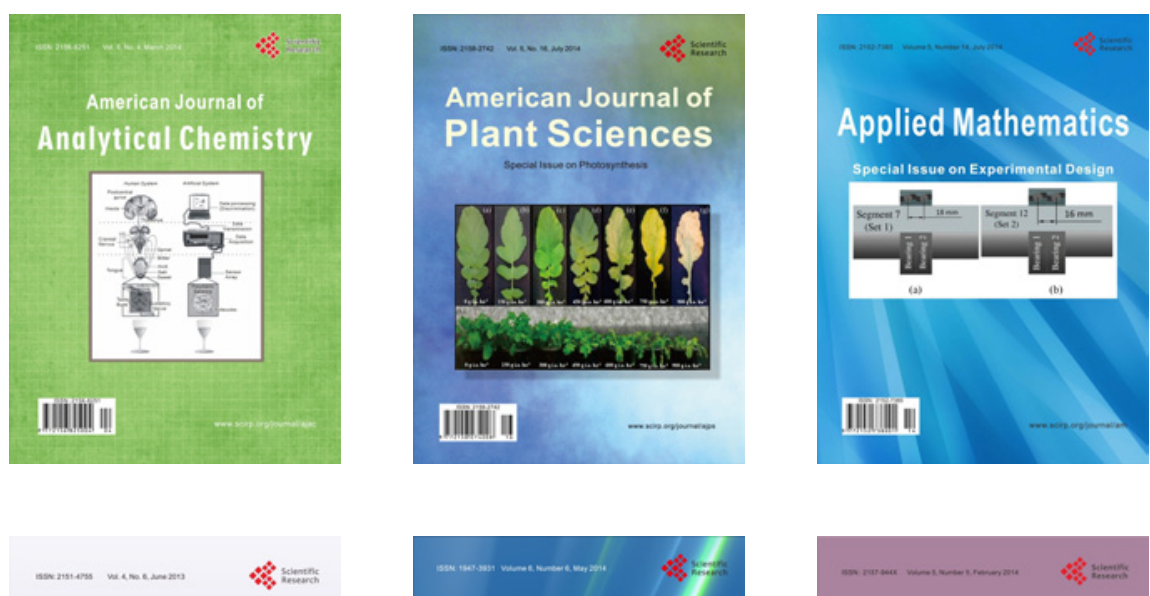

Creative Education
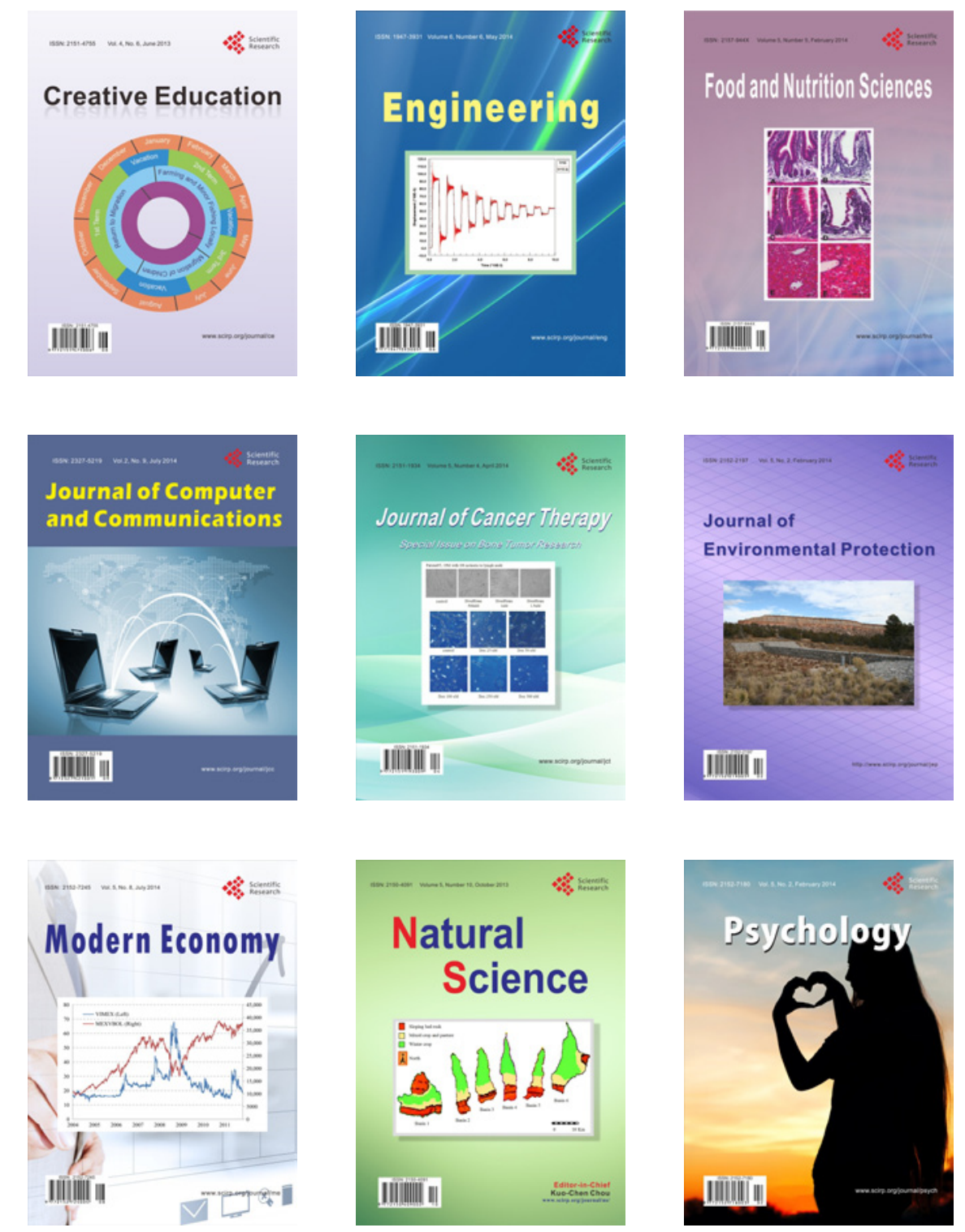